\title{
The COST259 Directional Channel Model-Part I: Overview and Methodology
}

\author{
Andreas F. Molisch, Fellow, IEEE, Henrik Asplund, Member, IEEE, Ralf Heddergott, Member, IEEE, \\ Martin Steinbauer, Member, IEEE, and Thomas Zwick, Senior Member, IEEE
}

\begin{abstract}
This paper describes a model for mobile radio channels that includes consideration of directions of arrival and is thus suitable for simulations of the performance of wireless systems that use smart antennas. The model is specified for 13 different types of environments, covering macro- micro- and picocells. In this paper, a hierarchy of modeling concepts is described, as well as implementation aspects that are valid for all environments. The model is based on the specification of directional channel impulse response functions, from which the impulse response functions at all antenna elements can be obtained. A layered approach, which distinguishes between external (fixed), large-scale-, and small-scale- parameters allows an efficient parameterization. Different implementation methods, based on either a tapped-delay line or a geometrical model, are described. The paper also derives the transformation between those two approaches. Finally, the concepts of clusters and visibility regions are used to account for large delay and angular spreads that have been measured. In two companion papers, the environment-specific values of the model parameters are explained and justified.
\end{abstract}

Index Terms-Direction of arrival, mobile radio channel, smart antennas.

\section{INTRODUCTION}

$\mathbf{S}^{\mathrm{T}}$ TANDARD models for mobile radio channels are important tools for the development of new radio systems and technology. They allow estimation of the benefits of different multiple access techniques, signal processing, and other measures for enhancing the capacity and improving performance, obviating the necessity to build a hardware prototype for every system under consideration, and test it in the field. A wellknown example of such an approach was the specification of the Global System for Mobile Communication (GSM) system [1], which relied on wideband channel models derived within the European research initiative COST (Cooperation

Manuscript received May 15, 2001; revised May 15, 2003; accepted February 17, 2005. The associate editor coordinating the review of this paper and approving it for publication was P. Driessen. Part of this work was supported by an INGVAR grant of the Swedish Strategic Research Foundation (SSF)

A. F. Molisch was with the Institut für Nachrichtentechnik und Höchfrequenztechnik (INTHF) of the TU Wien, Vienna, Austria and with AT\&T Labs-Research, Middletown, NJ, USA. He is now with Mitsubishi Electric Research Labs, Cambridge, MA USA, and also at Lund University, Lund, Sweden (e-mail: Andreas.Molisch@ieee.org).

H. Asplund is with Ericsson Research, Stockholm, Sweden (e-mail: Henrik.Asplund@ericsson.com).

R. Heddergott was with ETH Zurich, Zurich, Switzerland. $\mathrm{He}$ is now with Siemens Corporate Research, Munich, Germany (e-mail: ralf.heddergott@siemens.com).

M. Steinbauer was with the INTHF of the TU Wien, Vienna, Austria. $\mathrm{He}$ is now with mobilkom, Austria (e-mail: M.Steinbauer@mobilkomaustriagroup.com).

T. Zwick was with the Institut für Höechstfrequenztechnik und Eletronik, Universität Karlsruhe, Germany, and with the IBM T. J. Watson Research Center, Yorktown Heights, NY USA. He is now with Siemens AG, 88131, Lindau, Germany (email: Thomas.Zwick@web.de).

Digital Object Identifier 10.1109/TWC.2006.01117 in Science and Technology) 207 [2]. In addition, for system conformance tests, channel models (implemented in a channel simulator) are essential to guarantee reproducibility of results.

COST 259 was a European research initiative in the field of "Flexible Personalized Wireless Communications," which encompassed representatives of the major manufacturers, network operators, and many universities. Recognizing the importance of smart antennas and channel models that allow their realistic evaluation, it set as one of its aims the development of such a model.

Models for mobile radio channels must fulfill conflicting requirements. On one hand, they should be detailed enough to reflect all relevant properties of propagation channels; they should also not be misleading, and relevant limitations should be explained to prevent misapplication. On the other hand, they should be simple enough to allow rapid implementation and short simulation times. Channel models developed in the past have therefore naturally concentrated on those aspects that were important at the time they were developed. Consequently, most existing models give only field strength values (like Okumura-Hata [3], Lee's model [4], or COST231 Walfish-Ikegami [5]), which are of primary importance for narrowband systems, or power delay profiles (COST207 GSM [2], International Telecommunications Union (ITU)-R [6], and IEEE 802.11a/b [7]), which are of equal importance for second-generation digital wideband systems. However, in third-generation systems like Universal Mobile Telecommunications System (UMTS) [8] or IEEE 802.11n, as well as in fourth-generation systems, smart antennas will be a key technology. Channel models that include consideration of directions of arrival are required for simulation and testing of such systems.

There have already been several suggestions for directional channel models. ${ }^{1}$ General modeling methodologies have been presented both for spatial wide-sense stationary uncorrelated scattering (WSSUS) [9] and geometrically-based approaches [10], [11], but these do not contain detailed parameterization for different environments. For indoor channels, the Saleh-Valenzuela model [12] has been generalized to include directional information in [13]. Similarly, [14] adds directional information based on measurements for outdoor environments in order to generalize the ITU-R models, while [15] uses theoretical considerations in order to generalize the COST207 model. The authors of [16] report a stochastic approach, and suggests a simple model for the angle-of-arrival distribution based on measurements in rural and regular urban structures; [17] suggest the use of the von Mises distribution

\footnotetext{
${ }^{1}$ Channel models that are suitable for simulations with smart antennas were recently introduced for $3 \mathrm{GPP}$ and $802.11 \mathrm{n}$. These models make use of a number of concepts developed in COST259.
} 
for the angles-of-arrival. The papers [18], [19] propose a geometrical approach, and suggest some parameterization for outdoor environments. The papers [20] and [21] geometrically model the waveguiding effect near street canyons. Models reported in the above-cited literature give valuable insights into propagation effects and their effect on smart antenna systems. However, we see from the above description that the cited papers usually model only a few parameters (we will see below that a considerable number of parameters is needed to completely characterize propagation channels), without giving their interrelationships with other parameters. More specifically, it is only the angular spread in one small area that is described, without taking into account a variation in this and other associated parameters for different locations of the MS in different parts of a cell. A further problem is the possible statistical non-stationary properties of the channel process [22], e.g. when a mobile turns around a corner, and new propagation paths suddenly become possible. ${ }^{2}$

Thus, COST259 set out to develop a channel model with the following objectives:

- Agreement with measurements: considering that there is an increasing number of reported measurements for many environments, an objective was established that the statistics of measured impulse response estimates should be reproduced. The COST 259 model reproduces many of the characteristics obtained from measurement campaigns; for a more detailed discussion see [24].

- Consistency: in order to build on the knowledge from preceding COST projects like COST207, it was also set as an objective that the model should be made backwardcompatible by incorporating the old model constructs as special realizations. The COST 259 Directional Channel Model (DCM) fulfills this criterion, as described in [23].

- Simplicity: it was established that the model should be formulated in such a way that all effects are modeled by a very simple approach. This renders mathematical tractability and low computational complexity. As the model is more general than, e.g., the COST207 model, it is also more complex. However, it is quite feasible to implement within reasonable time, and several computer (Matlab) implementations are now in use throughout the world.

- Completeness; an objective was that macro- micro- and picocells should be modelled. Frequencies from $800 \mathrm{MHz}$ up to $2 \mathrm{GHz}$ (for outdoor) and $800 \mathrm{MHz}$ up to $5 \mathrm{GHz}$ (for indoor) should be covered to allow modeling for both present and future systems. All propagation effects that are relevant to the performance of the system should be included.

The objective of accuracy and simplicity are sometimes contrary. Thus, a good trade-off has to be made in the design of the model.

The present paper, and its companion papers [24], [25] (henceforth called Paper II and Paper III) give a complete description of the COST259 DCM, using a mixed deterministic/stochastic description of mobile radio channels in different radio environments. The present paper starts with

\footnotetext{
${ }^{2} \mathrm{~A}$ number of additional papers have been published since the submission of this manuscript.
}

TABLE I

ACRONYMS

$\begin{array}{ll}\text { BS } & \text { Base Station } \\ \text { CIR } & \text { Channel Impulse Response Function } \\ \text { COST } & \text { Cooperation in Science and Technology } \\ \text { DCIR } & \text { Directional Channel Impulse Response Function } \\ \text { DCM } & \text { Directional Channel Model } \\ \text { DDCIR } & \text { Double-Directional Channel Impulse Response Function } \\ \text { DOA } & \text { Direction of Arrival } \\ \text { DOD } & \text { Direction of Departure } \\ \text { GP } & \text { Global Parameters } \\ \text { GSCM } & \text { Geometry-based Stochastic Channel Model } \\ \text { IO } & \text { Interacting Object } \\ \text { LOS } & \text { Line of Sight } \\ \text { LP } & \text { Local Parameter } \\ \text { MPC } & \text { MultiPath Component } \\ \text { MPG } & \text { MultiPath Group } \\ \text { MS } & \text { Mobile Station } \\ \text { NLOS } & \text { Non Line of Sight } \\ \text { PAS } & \text { Power Angular Spectrum } \\ \text { PDDP } & \text { Power Delay Direction Profile } \\ \text { pdf } & \text { probability density function } \\ \text { PDP } & \text { Power Delay Profile } \\ \text { PS } & \text { Propagation Scenario } \\ \text { RE } & \text { Radio Environment } \\ \text { RF } & \text { Radio Frequency } \\ \text { RX } & \text { Receiver } \\ \text { TX } & \text { Transmitter } \\ \text { VDA } & \text { Virtual Deployment Area } \\ \text { WSSUS } & \text { Wide-Sense Stationary Uncorrelated Scattering } \\ \end{array}$

a description of the basic methods that can be used for channel simulation, namely deterministic approaches, as well as stochastic methods that might or might not need the WSSUS [26] assumption. We also give a system-theoretic description of propagation channels and a general definition of the quantities we wish to describe. A multilayered modeling approach is used, as it allows channel parameters to be easily changed on different scales in accordance with observations from measurements. We thus define "global" and "local" parameters that are characterized statistically. Next, we define a set of environments for macro-micro- and picocells. These "standard" environments are characterized by certain external (constant) parameters, but also influence the probability distributions of the global and local parameters. Finally, we consider the fact that multipath components (MPCs) often arrive in clusters, and discuss various ways in which this can be exploited to simplify simulation procedures. Papers II and III discuss the explicit values for external, global, and local parameters as well as their justification from measurements.

The following notation conventions will be adhered to: We denote matrices by underlined variables, and vectors by an overhead arrow. $\langle\cdot, \cdot\rangle$ is the scalar product in $\mathcal{R}^{3}$

\section{The Modelling OF Dispersion}

\section{A. Physical Properties of Mobile Radio Channels}

On a radio link in a cluttered environment the energy radiated by the transmitter (TX) most often reaches the receiver (RX) by traveling over multiple paths. Along these paths, interactions may occur between the electromagnetic field and various objects. Some possible interactions include specular reflection on large plane surfaces, diffuse scattering from surfaces exhibiting small irregularities or from objects of small size, transmission through dense material, diffraction, and shadowing by obstacles. The adjectives "large" and "small" are to be understood with respect to the wavelength. In the 
following, all objects that interact with the electromagnetic field so as to have non-negligible influence on the received signal are called "interacting objects" (IOs).

What is referred to herein as propagation scenario is dependent upon, and defined by, the physical positions of the TX and the RX as well as by the positions of IOs and their electromagnetic properties. At the RX antenna, multipath propagation leads to a superposition of waves or MPCs, each of which is assumed herein as a plane wave with a different delay, direction of arrival, amplitude, phase, and polarization. The corresponding delay dispersion and direction dispersion cause frequency selectivity and spatial selectivity, respectively [27]. The plane wave assumption is an idealization commonly used in Radio Frequency (RF) engineering, as any electromagnetic field can be decomposed into an (infinite) number of plane waves. If only a finite number of plane waves is used, the assumption is usually not fulfilled exactly; the error depends (among other things) on the distance between the wave sources and the TX and RX antennas.

In propagation scenarios, the parameters of impinging MPCs can be regarded as constant for displacements of the mobile station (MS) within a range of a few wavelengths (except for the phase). Such a range is called local area. Criteria that must be fulfilled within such an area include the narrow-band assumption [28], and the far-field assumption [29]. Furthermore, a local area must be fully contained within the "local region of stationarity" as defined in [30]. Consideration of these criteria indicates that the size of a local area is dependent on the carrier frequency and the system bandwidth, as well as the minimum distance of the RX antenna to the next radiating source (the TX antenna or an IO) [31]. Movements of the MS within a local area give rise to small-scale fluctuations, which occur due to changes in the phase relations between the MPCs. Displacements of the MS exceeding the size of a local area result in considerable variations of the delay, incidence direction, and amplitude of the MPCs; moreover, MPCs may vanish or new MPCs can be received [32]. The corresponding effects are denoted as large-scale fluctuations. A mathematical definition of these ideas will be given below.

\section{B. The Directional Channel Impulse Response Function}

In the COST259 DCM, the dispersion characteristics of a propagation channel in delay and angle of incidence are described by the directional (or double-directional) impulse response function of the channel, an equation that will be derived in the sequel. We start by describing the properties of an arbitrary (index $\ell$ ) MPC, which is assumed to be a plane wave. It is assumed to have been received over a propagation path that can be modelled using the double directional impulse response function

$$
\underline{h}_{\ell}\left(\overrightarrow{r_{0}}, \tau, \Omega, \Psi\right)=\underline{a}_{\ell} \delta\left(\tau-\tau_{\ell}\right) \delta\left(\Omega-\Omega_{\ell}\right) \delta\left(\Psi-\Psi_{\ell}\right),
$$

where $\tau$ is the delay variable and the spatial angle $\Omega$ characterizes the direction of arrival (DOA) of waves at the RX antenna. $^{3}$ The direction of departure (DOD) of waves from the TX antenna is denoted by $\Psi$. Moreover, $\underline{\alpha}_{\ell}$ denotes

\footnotetext{
${ }^{3}$ The spatial angle $\Omega$ corresponds to a point on the unit sphere. Note that when it is represented by its azimuth angle $\phi$ and its elevation angle $\vartheta$, an integration over $\Omega$ requires the inclusion of the Jacobian, $\mathrm{d} \Omega=\cos \vartheta d \vartheta d \phi$.
}

(complex) amplitude. Note that the impulse response function is a polarimetric $2 \times 2$ matrix, as is the complex amplitude $\underline{\alpha} \ell$

$$
\underline{\alpha}_{\ell}=\left(\begin{array}{cc}
\alpha_{\ell}^{\vartheta \vartheta} & \alpha_{\ell \phi}^{\vartheta \phi} \\
\alpha_{\ell}^{\phi \vartheta} & \alpha_{\ell}^{\phi \phi}
\end{array}\right)
$$

where superscript $\vartheta$ and $\phi$ denote polarization in $\vartheta$ and $\phi$, respectively (due to the far-field assumption, two orthogonal polarizations are sufficient for the characterization). Within a local area $A$, it is assumed that the variation of the propagation delay, attenuation, and angle-of-incidence of the impinging waves due to modification of the propagation path lengths as a result of MS motion can be neglected. ${ }^{4}$ Therefore, in (1), $\vec{r}_{0}$ denotes an arbitrary reference point within $A$. Furthermore, $\vec{r}$ denotes the location of the RX antenna with respect to the TX antenna. $^{5}$

With the position of the TX fixed at the origin of the coordinate system, and the RX at any position within the local area, the equivalent impulse response function of a particular path $h_{\ell}(\vec{r}, \tau, \Omega, \Psi)$ between the TX and RX can be written as

$$
\underline{h}_{\ell}(\vec{r}, \tau, \Omega, \Psi)=\underline{h}_{\ell}\left(\vec{r}_{0}, \tau, \Omega, \Psi\right) e^{j \frac{2 \pi}{\lambda}\left\langle\vec{e}\left(\Omega_{\ell}\right), \vec{r}-\vec{r}_{0}\right\rangle} .
$$

The phase change is computed by the location-dependent part of the plane-wave exponential factor $e^{j \frac{2 \pi}{\lambda}\left\langle\vec{e}\left(\Omega_{\ell}\right), \vec{r}-\vec{r}_{0}\right\rangle}$. Herein, $\vec{e}(\Omega)$ denotes a unit vector pointing towards $\Omega$.

At the position of the RX antenna, different MPCs (with different parameters) are incident. The double-directional channel impulse response function (DDCIR) can thus be written as the sum of the contributions from $L$ different waves ${ }^{6}$

$$
\begin{aligned}
& \underline{h}(\vec{r}, \tau, \Omega, \Psi)=\sum_{\ell=1}^{L(\vec{r})} \underline{h}_{\ell}(\vec{r}, \tau, \Omega, \Psi) \\
= & \sum_{\ell=1}^{L(\vec{r})} \underline{h}_{\ell}\left(\vec{r}_{0}, \tau, \Omega, \Psi\right) e^{j \frac{2 \pi}{\lambda}\left\langle\vec{e}\left(\Omega_{\ell}\right), \vec{r}-\vec{r}_{0}\right\rangle} .
\end{aligned}
$$

We have written the impulse response function here as a finite sum of equivalent impulse response functions for the individual paths over which waves are received at a given location. This is valid if only MPCs whose amplitudes are above a certain threshold (e.g., the noise level) are considered. Otherwise, the summation has to be replaced by an integration. We assume that the IOs are stationary, so that the position $\vec{r}$ is related to absolute time $t$ by the trajectory of the MS. Consequently, we can replace the notation $h(\vec{r})$ henceforth by the more common $h(t)$. The implications of assuming stationary IOs are discussed later.

The above description is a characterization of a channel in isolation. When filtered by a $\mathrm{RX}$ with a finite bandwidth $B$, and thus limited resolution capability in the delay domain, the interpolating function (suppressing the other arguments of the

\footnotetext{
${ }^{4}$ The following description assumes a moving receiver. As the transmitter is the point of origin of the coordinate system, this is no restriction of generality.

${ }^{5}$ Note that the position $\vec{r}$ and the angle of incidence at the receiver $\Omega$ form a Fourier transform pair [27]. Still, we write a dependence of $h$ on both these variables, as this is more intuitive.

${ }^{6}$ The summation is admissible if the channel is linear. This implies that the scattering objects, as well as the antennas, should not contain nonlinear or gyromagnetic materials.
} 
function) is [26]

$$
\underline{h}(\tau)=\sum_{\ell=1}^{L(\vec{r})} \underline{a}_{\ell} \operatorname{sinc}\left(B\left(\tau-\tau_{i}\right)\right) .
$$

Depending on the bandwidth, several MPCs can be contained within each delay bin of width $1 / B$, and interfere constructively or destructively (depending on their phase relationships), thus giving rise to fading when the TX and/or RX moves.

The DDCIR can be related to the directional channel impulse response function (DCIR), which is the quantity of interest when there are multiple-antenna elements at only one end of the radio link under study (without restriction of generality we assume that they are at the RX). The DCIR is obtained by integration of $h(t, \tau, \Omega, \Psi)$, weighted by the complex polarimetric antenna pattern $\vec{G}_{T X}(\Psi)$ of the TX antenna, over direction: ${ }^{7}$

$$
\vec{h}(t, \tau, \Omega)=\int_{\Psi} \underline{h}(t, \tau, \Omega, \Psi) \vec{G}_{T X}(\Psi) \mathrm{d} \Psi .
$$

Similarly, the DCIR can be related to the non-directional time-variant channel impulse response function $(\mathrm{CIR})^{8}$ by integrating over the DoAs

$$
h(t, \tau)=\int_{\Omega} \vec{h}(t, \tau, \Omega) \vec{G}_{R X}(\Omega) \mathrm{d} \Omega .
$$

To simplify notation, we will in the following refer to one component of the directional full-polarimetric CIR, namely the $\vartheta$-polarized component, as $h(t, \tau, \Omega)$, and this is a scalar function. Due to the number of arguments, there is no danger of confusing it with the non-directional CIR $h(t, \tau)$. In the remainder of this paper and in Papers II and III, we will deal with this quantity as the directional CIR if not explicitly stated otherwise. Also, whenever presenting simulation results for DCIRs or non-directional CIRs, we assume omni-directional antenna patterns.

With these definitions, we can also formalize the notion of local area. The parameters of the incident waves, $\alpha_{\ell}, \tau_{\ell}, \Omega_{\ell}$, do not change for movement of the MS within a local area $A$. Within such an area, the set of (constant) parameters

$$
\left\{L(\vec{r}), \alpha_{1}, \tau_{1}, \Omega_{1}, \ldots, \alpha_{L}, \tau_{L}, \Omega_{L}\right\} .
$$

characterizes a channel's impulse response function, together with the probability density functions (pdfs) for the phases $p d f_{\beta_{1}, \ldots \ldots . \beta_{L}}\left(\beta_{1}, \ldots . \beta_{L}\right)=1 /(2 \pi)^{L}$.

For future use, we specify a (small-scale) power delaydirection profile (PDDP)

$$
P_{A}(\tau, \Omega)=\mathrm{E}_{\vec{r} \in A}\left\{|h(\vec{r}, \tau, \Omega)|^{2}\right\} .
$$

where $\mathrm{E}_{\vec{r} \in A}\{\cdot\}$ denotes the expectation over all locations within $A$. The generation of impulse response functions from a specified small-scale PDDP will be treated in Sec. IV.B.1. We also define the power delay profile (PDP) as $\int P_{A}(\tau, \Omega)\left|G_{R X}(\Omega)\right|^{2} \mathrm{~d} \Omega$, and the power angular spectrum

\footnotetext{
${ }^{7}$ The frequency dependence of the antenna pattern is neglected henceforth.

${ }^{8}$ Strictly speaking, this function is not the response of the channel at a certain time instant, but the response at the Rx antenna output at time $t$ if a Dirac impulse was transmitted at time $t-\tau$. Bello [26] has denoted $h(t, \tau)$ input delay spread function.
}

(PAS) as $\int P_{A}(\tau, \Omega) \mathrm{d} \tau$. The summing up of the powers coming from different directions entails the assumption that these contributions are uncorrelated. This assumption is equivalent to the "extended WSSUS" assumption, which is discussed, e.g., in [33], and [9].

\section{Stationary IOs}

The assumption that only the MS moves, and the IOs are stationary, is an important restriction of the model, especially in the context of directional measurements and modeling. It is very useful as it allows a much simpler modeling and simulation procedure; however, one has to be aware of the implied restrictions. Directionally and/or delay-resolved systems allow to distinguish between different IOs. When only some IOs (e.g., cars) move, the relative distance between MS and only the moving IOs changes. When only the MS moves, the relative distance between MS and all IOs changes. This also happens when both MS and IOs move; however, the relationship between the phase changes are different. Furthermore, when only the MS moves, the DOAs at the MS (plus the velocity vector of the MS) uniquely determine the Doppler spectrum; this is not the case when the IOs move.

The applicability of the assumption of stationary IOs depends on the considered scenarios. For macrocells, the signals propagate mainly via building, mountains, and other stationary structures [34]. However, there may be significant scattering by cars and pedestrians near the MS. Even for networks that were planned so as to minimize temporal variations on their constituent radio links, fading that can be well modelled using Rician envelope fading distributions, with Rice factors as low as $10 \mathrm{~dB}$ has been reported [35]. In micro- and picocells, the influence of pedestrians might be even larger, as many of the dominant propagation processes (see Paper III) occur at the height of pedestrians and/or cars. A further complication is introduced by the fact that some of those IOs might not just change the small-scale statistics, but could also act as shadowing objects.

The effect of moving IOs can thus be twofold:

- if the IOs are far away, their movement leads to fading of those MPCs that are associated with those IOs. As directional and delay-resolved systems can distinguish between different IOs, the fading is more pronounced in the directions (at the delays) of the moving IOs. The impact on system performance depends on the strength of the MPCs created by the moving IOs compared to the strength of all other components, as well as their angles of incidence.

- IOs that are moving close to the MS can shadow other MPCs. This effect can be modeled geometrically, e.g., by appropriate attenuation of all MPCs coming from an angular range; this angular range is determined by the distance and the size of the objects. In order for this effect to be negligible, it is necessary that the total power of the shadowed components is much smaller than the total power.

\section{Modeling Constructs And Their Hierarchy}

\section{A. Radio Channel Simulation Methods}

Four methods have been proposed for the simulation of the radio channel impulse response functions (CIRs): 
1) Stored CIR's are by definition the most accurate and realistic CIRs. On the other hand, it is very difficult to define a set of stored CIRs that is typical for a certain environment and includes all relevant effects. If an exhaustive set of CIRs is used, storage requirements become excessive. Furthermore, such stored measurement results are rarely shared openly.

2) Ray optical methods are a very powerful tool to perform a coverage prediction in a particular propagation scenario. Ray tracing results are easier to obtain than measured CIRs. The main drawback is the requirement for a very precise database, and an exact definition of the propagation effects that are taken into account.

3) Stochastic radio channel models describe the CIR as realizations of a stochastic process. The WSSUS-model [26] characterizes propagation channels by means of correlation functions or power spectral densities. The low-pass envelope equivalent impulse response of a radio channel is represented by a tapped delay line, where each tap represents the fading resultant of the vector addition of a number of unresolvable MPCs. Ref. [36] also suggested that the spectral components of the channel transfer function could exhibit (possibly correlated) complex Gaussian amplitude statistics, in that case, the model should be referred to as the Gaussian WSSUS (GWSSUS) model. The absolute amplitudes then follow a Rayleigh or Rician statistics, depending on whether the complex amplitudes have zero mean or not. Later papers have suggested that also the taps of a tapped delay line representation might exhibit complex Gaussian fading (for a discussion of that assumption, see Sec. V). The stochastic modeling approach is, for example, used in the COST207 model [2].

4) Parametric stochastic models [37] use a different methodology for the definition of probability distribution functions. Incident waves at the receiver are described not by their correlation functions, but rather by certain parameters, such as delay and DoA. These parameters remain constant for movements of the MS over a distance of a few tens of wavelengths. The WSSUS property is not required. The parametric approach is the one used for most parts of the COST 259 DCM. ${ }^{9}$

\section{B. Propagation Scenarios}

Radio propagation depends on topographical and electromagnetic features of the operating environment. To account for variations in these characteristics, a 3-level structure according to Fig. 1 has been defined for the COST259 DCM. It provides a framework through which channel models that are most applicable to specific types of systems can be deduced.

At the top level, a first distinction has been made by the cell type, namely macro- micro- and picocells. For each cell type, a number of radio environments (RE's) have been identified, where all names begin with the word "generalized." This has been chosen to stress that a RE stands for a whole class, $\mathcal{R}$, of multipath conditions that give rise to similar radio channel characteristics that, in turn, can be related to the

\footnotetext{
${ }^{9}$ There are some deterministic/ray tracing components in the higher layers of the model hierarchy, as will become clear below.
}

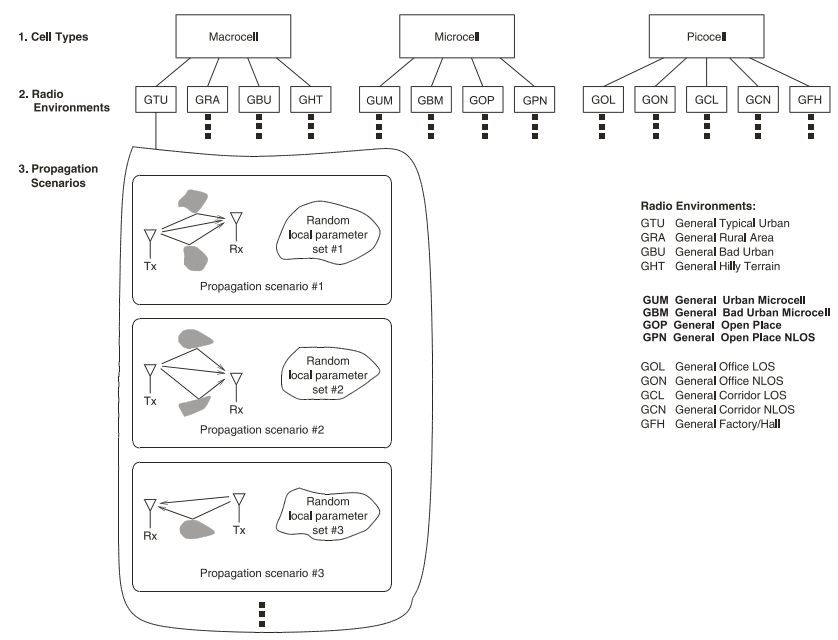

Fig. 1. Layered structure of the COST259 DCM.

surroundings in which a communication system operates. This is an extension of the COST 207 channel models, since each realization of the latter exhibits only the typical propagation conditions of a particular set of surroundings.

The bottom layer of the hierarchical structure of modeling constructs consists of the Propagation Scenarios (PS), which are defined as random realizations of multipath conditions (see Fig. 1). PSs are not classes in the strict mathematical sense but represent certain small-scale channel states each of which exhibits a constant PDDP and scattering function. ${ }^{10}$ Largescale parameters remain constant within a PS. This implies that a PS must reflect conditions within a limited physical area $A$ as discussed in Eq. 8. ${ }^{11}$

\section{Parameter Types}

The features of a RE are defined by a number of external parameters, such as the frequency band, the average height of Base Station (BS) and MS antennas, their average distance from each other, and average building heights and separations. In many REs, conditions of line-of-sight (LOS) or non-lineof-sight (NLOS) are specified. In other REs both might occur on a stochastic basis.

The propagation conditions of each RE are described by a set of fixed parameters, as well as probability density functions for stochastic parameters and PDDPs. Since they characterize the propagation conditions of the entire radio environment, they are called global parameters (GPs). ${ }^{12}$ An example for a

\footnotetext{
${ }^{10}$ Note that the individual realizations of the CIRs have different phases of the MPCs. It is therefore possible to define a scattering function or equivalent representation that uses an expectation over the CIRs, even though the magnitudes and delays of the associated MPCs are constant.

${ }^{11}$ In the past, "local areas" have often been defined as those systems where the "mean propagation loss" is constant. If the mean pathloss is to be computed from measured (or simulated) spatial samples of the CIR, the variance of the result has to be small so that it can be tested whether the pathloss is constant. This implies that there has to be a sufficient number of independent samples, so that averaging over a certain area is required. The pathloss in turn is dependent on the size of the required averaging area. The definition Sec. II.C, Eq. (7) does not show this dependence, since it considers the change of parameters of the multipath parameters, which do not depend on averaging lengths. However, the extraction of those values from measurement results often requires an a-priori assumption that the multipath parameters do not change over the size of a measurement array.

${ }^{12}$ Note that "global" parameters are different in different radio environments.
} 
GP is the number of visible IO clusters, which is characterized by a Poisson distribution ${ }^{13}$ with parameter $\lambda$ (note that both the shape of the distribution function and the parameter $\lambda$ are GPs). GPs serve as key parameters that provide the necessary information for system design decisions on modulation technique, burst length, coding scheme, etc. They must be extracted from detailed measurement data of statistically significant quantity. Recently, ray tracing simulations have also been used for GP extraction [38]. Propagation scenarios are controlled by the GPs of the associated RE.

Local parameters (LPs) are random realizations of parameters that describe instantaneous channel conditions in a local area. The statistical properties of the LPs are given by the set of GPs defined in the 2nd (medium) level of the COST259 DCM hierarchy. Note that global and local parameters can depend on the external parameters. It is considered reasonable to assume that for movements of the MS within a sufficiently small local area A, not larger than some tens of wavelengths, the LPs determining the propagation scenario remain approximately constant. As a consequence, the spatial variations of a CIR within a local area are modeled as changes in the phases of the impinging waves. Since with a finite bandwidth it is not possible to resolve all MPCs, the phase variations of the impinging waves caused by motion result in rapid fluctuations of amplitudes and phases of the multipath groups within a CIR. These fluctuations are called small-scale fluctuations.

\section{Cell Types}

The first and essential distinction is between macro- microand picocells. Macrocells are characterized by cellsizes in the km-range, and a marked difference between the height of BS and MS antennas, as the BS antenna is, by definition, placed above the height of surrounding rooftops. Thus, the immediate surroundings of BS and MS have different characteristics, leading to different DoA pdfs. In micro- and picocells, on the other hand, BS and MS are mostly co-located in the same environment of IOs and can be associated with similar DoA distributions.

Micro- and picocells are (by definition for this model) distinguished by the fact that picocell BSs are located indoors, while for microcells, they are located outdoors. In the scenarios described by the COST259 DCM, the microcell MS is also outdoors, while the picocell MS is indoors. Thus, "penetration" scenarios (coverage of a building by an outdoor BS, or coverage of an office courtyard by an indoor BS) are not specified explicitly.

In macro-, micro-, and picocells, both LOS and NLOS situations can occur. LOS or quasi-LOS operation occurs more often in the microcells modeled in COST 259. Microcells are currently employed mainly in urban areas with high user density, which induces a large capacity demand, i.e., in city centers. ${ }^{14}$ Suburban and rural environments, where large areas need to be covered by few BSs, mainly use macrocells, possibly equipped with smart antennas.

\footnotetext{
${ }^{13}$ This is based on the assumption that the process of the MS being present in a visibility region can be modeled as a Poisson process.

${ }^{14}$ American environments might be different, as high traffic density might occur more in (suburban) shopping malls and entertainment centers.
}

\section{E. Radio Environments}

The medium layer of the hierarchical structure of the COST259 DCM is occupied by Radio Environments. The subdivision of each of the cell types into REs by morphological characteristics is by its very nature somewhat arbitrary. As in all approaches to classification, there is a tradeoff between accuracy (which could lead to the definition of hundreds of REs, each with slightly different parametrization), and simplicity. Our goal was to define no more than 15 REs, to allow access to a simulation model that can represent a range of cases without necessitating excessive amounts of computation or measurements.

1) Macrocells: For macrocells, the motivation for subdivision into typical urban, bad urban, rural area, and hilly terrain categories was to keep a degree of compatibility with the COST 207 model. We also note that for macrocells, the appearance and disappearance of line-of-sight (LOS) is handled statistically - in contrast to the micro-and picocells, see below. We thus define four different macrocell REs, including

- The generalized Typical Urban RE, denoted GTU.

- The generalized Bad Urban RE, denoted GBU.

- The generalized Rural Area RE, denoted GRA.

- The generalized Hilly Terrain RE, denoted GHT.

Detailed descriptions of these environments can be found in Paper II.

2) Microcells: For microcells the REs are much smaller in size than for the macrocells. Since the BS antenna is below rooftop, the basic propagation characteristics change on a much smaller scale than in a macrocell. We have defined:

- The generalized Urban Microcell, denoted GUM, as the typical situation where both the BS and the MS are in a street canyon or on a street crossing.

- The generalized Bad Urban Microcell RE, denoted GBM, which is similar to the GUM, but with high-rise buildings in the surroundings.

- The generalized Open Place RE, denoted GOP, in which both BS and MS are in an open area. A line-of-sight signal is usually received as well as delayed MCs that result from interactions with IOs "visible" from the MS, e.g. along the perimeter of the open area.

- The generalized Open Place Non-line-of-sight RE, denoted GPN, in which either the BS or the MS is on the open area, while the other one is in a street canyon or a nearby street crossing.

3) Picocells: The subdivision of REs in the picocell environment follows a conceptualization similar to that associated with microcells. It is intuitively clear that a corridor, which exhibits waveguiding effects, is fundamentally different from a room. Also, LOS and NLOS situations are treated as different REs. Finally, a factory hall or lounge must be treated as a separate environment, since the geometrical dimensions are so much larger than those of a standard (office) room, so that different rms delay spreads are to be anticipated. Picocell REs include:

- The generalized office LOS RE, denoted GOL, where both BS and MS are in the same large office or conference/meeting room with LOS between them. Such a room could be subdivided by lightly constructed walls or 


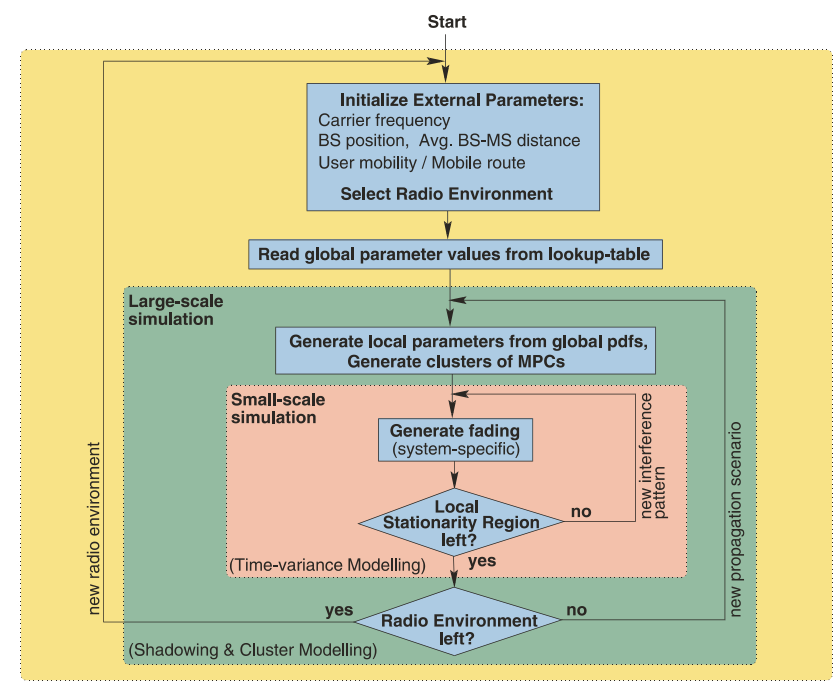

Fig. 2. Flowchart for the implementation of the COST259 DCM.

partitions, in which case there would be obstructed LOS (OLOS), see Paper III.

- The generalized Small Office NLOS RE, denoted GON, which is defined by a situation where a MS is in a small office, and the BS is mounted on the wall of a corridor leading to the office room.

- The generalized Corridor LOS RE, denoted GCL, which reflects a situation in which the MS and the BS are in the same corridor and have LOS to each other.

- The generalized Corridor NLOS RE, denoted GCN, which categorizes a situation in which the MS is within a corridor from which there is an obstructed view to the BS.

- The generalized Factory/Hall RE, denoted GFH, which describes "large open indoor areas." Note that a factory hall usually contains more metallic objects than other environments, which leads to a larger number of MPCs, and a less pronounced clustering structure than an event hall/auditorium. Still, the general characteristics of assembly halls and factories are similar enough to warrant a merging of such surroundings for classification by the same RE type.

\section{IMPLEMENTATION CONSIDERATIONS}

\section{A. The Generation of CIRs}

The structure of the COST 259-DCM, consisting of cell types, REs, and random PSs, defines a framework from which the DCIR, mathematically described in Eq. (6), can be derived. This requires the definition of a particular set of LPs and their statistics by means of GPs. The latter definitions should be based on the propagation conditions encountered in a given RE.

The parameters of a DCIR can be defined by the specification of a delay-angle distribution for the incident waves [39], [40], or of IO configurations [41]. In the sequel, both approaches and a transformation between PDDPs resulting from the specification of a delay-angle distribution and IO geometry are briefly sketched. The particular values of local and global parameters used in the COST 259-DCM are given in Papers II and III.
1) The Definition of Local and Global Parameters Based on the Delay-Angle Distributions: For the implementation based on the delay-angle distribution of received MPCs, we have to distinguish between the generation of LPs and the creation of a complex impulse response function. In a first step, we have to generate a small-scale PDDP (or equivalently, a parameter set Eq. (8)), from large-scale information. This large-scale information can either be obtained from geometrical considerations (see below), or from the probability density function (pdf) for the number of impinging waves $f(L)$, the pdf of multipath arrivals $f\left(\tau_{\ell}, \Omega_{\ell}\right)$, and the pdf for the power of the $\ell$-th wave, given its delay and angle of arrival $f\left(\left|\alpha_{\ell}\right|^{2} \mid \tau_{\ell}, \Omega_{\ell}\right)$. Note that a large-scale PDDP

$$
P_{\mathcal{R}}(\tau, \Omega)=\mathrm{E}_{A \in \mathcal{R}}\left\{\frac{1}{P_{A}} P_{A}(\tau, \Omega)\right\},
$$

where the expectation is taken over the scenarios identified with their local area $A$ within the radio environment $\mathcal{R}$, does not give sufficient information for the generation of the directional impulse response functions with correct statistics.

The further use of the PDDP depends on the specified resolution. In the case that it is specified with such high resolution that each MPC is resolved, the PDDP describes the power (and thus absolute amplitude), as well as the angles and delays of all MPCs; in order to generate a single realization of the CIR, we just have to assign a random phase to each of the MPCs.

In the case that the PDDP is specified with a smaller resolution, it describes the statistics of resolvable multipath groups (MPGs). In this case, the PDDP is related to the CIRs as

$$
P_{A}(\tau, \Omega)=\sum_{\ell=1}^{L} P_{A_{\ell}}(\tau, \Omega)=\sum_{\ell=1}^{L} \mathrm{E}_{\vec{r} \in A}\left\{\left|h_{\ell}(\vec{r}, \tau, \Omega)\right|^{2}\right\} .
$$

where $\ell$ now indexes the MPGs rather than single MPCs. Different realizations of the CIR are then obtained by assigning random complex amplitudes to different MPGs (in the case of the GWSSUS model, the complex amplitudes would obey a complex Gaussian statistics). It is obvious that the underlying stochastic process is only an uncorrelated scattering (US) process as defined by Bello [42] if the equality

$$
\sum_{\ell=1}^{L} \mathrm{E}_{\vec{r} \in A}\left\{\left|h_{\ell}(\vec{r}, \tau, \Omega)\right|^{2}\right\}=\mathrm{E}_{\vec{r} \in A}\left\{|h(\vec{r}, \tau, \Omega)|^{2}\right\}
$$

holds. In this case, the local average of $h(\vec{r}, \tau, \Omega)$ yields uncorrelated components. In [43] it has been stated that the US assumption is not always appropriate at least for indoor channels.

2) The Definition of Local and Global Parameters Based on the IO Geometry: In geometrically-based stochastic channel models (GSCMs), the probability density function of the geometrical location of the IOs is prescribed [41], [44], [10]. For each channel realization, the IO locations are taken at random from this pdf and then the multipath delays and directions of arrival are computed by a simple ray-tracing approach, which assumes that only single specular reflections occur, so that each of the reflectors (IOs) is uniquely associated with one MPC. Of course, the pdf of IO locations has to be 
selected in such a way that resulting PDPs and PASs at the $\mathrm{RX}$ agree reasonably with the measured values.

An extension of the basic GSCM places IOs not only around the MS, but also includes consideration of distant groups of IOs (far IO clusters). This corresponds to groups of high-rise buildings or mountains that can work as efficient IOs especially if they have LOS both to the BS and the MS. An alternative implementation, called NSCS (nonuniform scattering cross section) uses a pdf of the IOs that is uniform throughout the whole relevant cell area, but employs a weighting of the cross sections of the IOs, which can change depending on the location of the MS. This not only corresponds better to physical reality, but also is more computationally efficient [45]. The GSCM principle allows a very efficient implementation, especially since small-scale and large-scale effects can be easily separated. Small-scale fading results from the interference of the waves from the IOs, the phases of which change when the MS moves over distances that are on the order of a fraction of a wavelength. The amplitudes of the MPCs stay constant over a local area, which is in accordance with the concepts outlined in Sec. 2. The incorporation of large-scale effects is realized by changing the power received from the IOs and the appearance and disappearance of IO clusters.

We finally note that it is possible to combine a geometrical generation of large-scale parameters with the delay/angle distribution based treatment of small-scale effects. This approach is actually quite computationally efficient [45].

3) Transforms Between Implementation Methods: The Delay-Angle-Distribution approach and the GSCM are two methodologies that can be transformed into each other by mathematical transformations [11], [46], [47].

\section{IO Distribution $\rightarrow$ PDP / PAS}

The power delay profiles (PDPs) and power angular spectra (PASs) calculated from a local interaction model must be in reasonable agreement with experimental results. In this section we demonstrate how to analytically determine the PDP and PAS from different probability distributions for IO locations. Such a transformation allows the comparison of the delay and angular distributions caused by the different IO geometries and this helps in determining the best agreement with measurements. Mathematically speaking, this relation is the transformation of the random variables $r$ and $\phi_{M S}$ (leading to IO coordinates $x_{s}$ and $y_{s}$ ) to random variables $\tau$ and $\phi_{B S}$.

The relationship between these random variables can be derived through geometrical considerations. Assuming only single interactions we can write

$$
\begin{gathered}
\phi\left(x_{s}, y_{s}\right)=\operatorname{atan}\left(\frac{\mathrm{y}_{\mathrm{s}}}{\mathrm{D}+\mathrm{x}_{\mathrm{s}}}\right), \text { and } \\
\tau\left(x_{s}, y_{s}\right)=\frac{\sqrt{x_{s}^{2}+y_{s}^{2}}+\sqrt{\left(D+x_{s}\right)^{2}+y_{s}^{2}}}{c},
\end{gathered}
$$

where the same notation as that in Fig. 3 has been used, and $c$ is the speed of light. The transformation is done using a standard mathematical formalism employing the Jacobian [48]

$$
\begin{array}{r}
p d f_{2}\left(\tau, \phi_{B S}\right)= \\
p d f_{1}\left(x_{s}\left(\tau, \phi_{B S}\right), y_{s}\left(\tau, \phi_{B S}\right)\right) \cdot J\left(\tau, \phi_{B S}\right),
\end{array}
$$

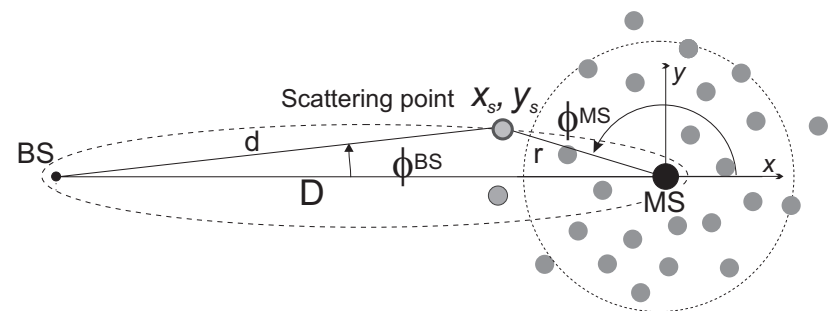

Fig. 3. Geometry for the transformation between scatterer and ADPS.

where $x_{s}\left(\tau, \phi_{B S}\right)$ and $y_{s}\left(\tau, \phi_{B S}\right)$ will be given below in Eqs. (17) and (18), respectively, and the Jacobian $J\left(\tau, \phi_{B S}\right)$ is

$$
\frac{(\tau c-D) \cdot(\tau c+D) \cdot\left(-2 \tau c D \cdot \cos \left(\phi_{B S}\right)+D^{2}+(\tau c)^{2}\right)}{4 \cdot\left(\tau c-\cos \left(\phi_{B S}\right) \cdot D\right)^{3}}
$$

\section{PDP / PAS $\rightarrow$ IO distribution}

We can also employ the same kind of transformations in the opposite direction. For example, the inverse transformation can be used to provide an IO geometry from a measured joint delay-angle distribution. This allows a straightforward adaptation of the GSCM to correspond to specific measured environments. After some manipulations the needed relationships between the random variables can be written as [47]

$$
\begin{gathered}
x\left(\tau, \phi_{B S}\right)=\frac{\left(D^{2}-(\tau c)^{2}\right)}{2 D-2 \tau c \sqrt{1+\tan ^{2}\left(\phi_{B S}\right)}}-D \\
y\left(\tau, \phi_{B S}\right)=\frac{\left(D^{2}-(\tau c)^{2}\right) \cdot \tan \left(\phi_{B S}\right)}{2 D-2 \tau c \sqrt{1+\tan ^{2}\left(\phi_{B S}\right)}}
\end{gathered}
$$

The pdf transformation employing the Jacobian can now be written

$$
p d f_{1}\left(x_{s}, y_{s}\right)=p d f_{2}\left(\tau\left(x_{s}, y_{s}\right), \phi_{B S}\left(x_{s}, y_{s}\right)\right) \cdot J\left(x_{s}, y_{s}\right),
$$

where $\phi_{B S}\left(x_{s}, y_{s}\right)$ and $\tau\left(x_{s}, y_{s}\right)$ are shown in Eqs. (13) and (14), respectively, and the Jacobian $J\left(x_{s}, y_{s}\right)$ is

$$
\left|\frac{\left(x^{2}+D x+y^{2}\right)+\sqrt{D^{2}+2 D x+x^{2}+y^{2}} \sqrt{x^{2}+y^{2}}}{\left(D^{2}+2 D x+x^{2}+y^{2}\right) \sqrt{x^{2}+y^{2}}}\right| .
$$

Note that the interaction model and the transformations described above are based on the assumption that only single interactions occur. However, recent measurements (e.g., [49]) have shown that at least in urban environments the observed propagation phenomena must be explained by multiple interactions. Thus, when we measure in a real-world environment and transform the joint delay-angle distribution to the corresponding spatial geometry assuming only single interactions, the resulting geometry does not, in general, fully agree with that of the real environment. However, when this pdf of the IO locations is used with the GSCM, the same delay and DoA 


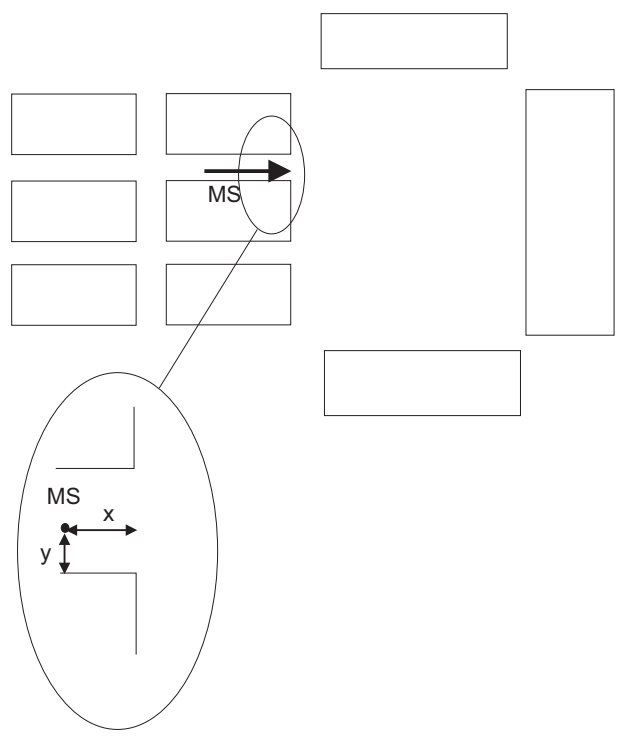

Fig. 4. Geometry for the computation of the transition function.

information can be reproduced at the base station, since the transformation is mathematically exact. ${ }^{15}$

\section{B. Transitions Between Radio Environments}

We next discuss how long a MS resides in one RE. In a macrocell, this duration is typically very long. In most cases a mobile will remain in the same RE for the complete duration of the call. For micro- and picocells, transitions from one RE to another (e.g. from a street to an open place; from an office out into a corridor) can easily occur during a period of interest for channel tracking applications; we therefore only consider these REs in the following. The transition is modeled by first generating two PDDPs, one according to the global parameters of the "old" RE, and one according to the global parameters of the "new" RE, i.e., the RE the MS moves into. We furthermore specify a transition function

$$
f_{\text {trans }}(x)=\frac{1}{2}-\frac{1}{\pi} \arctan \left(\frac{2 \sqrt{2} y}{\sqrt{\lambda|x|}} \operatorname{sign}(x)\right)
$$

where $x$ and $y$ are the distances of the antenna from the boundary between the REs, $x$ being the distance in the direction along the street (corridor) and $y$ being the direction normal to it (see Fig. 4). The PDDP at a location $x$ is then given as

$$
\begin{array}{r}
P_{A}(x ; \tau, \Omega)= \\
P_{A}^{R E 1}(\tau, \Omega) f_{\text {trans }}(x)+P_{A}^{R E 2}(\tau, \Omega)\left[1-f_{\text {trans }}(x)\right]
\end{array}
$$

The reason for choosing the function (21) is that it is an excellent approximation for the Fresnel integral with negative argument (i.e. in the shadowed region), but easier to compute. For positive arguments, the exact Fresnel integral shows oscillations that have rarely been observed in practice, whereas the function chosen in Eq. (21) is symmetrical with respect to the origin.

Actual values for the distances of the MS from the shadowing obstacles have to be chosen differently for the different

\footnotetext{
${ }^{15}$ Note that in this case, the transformation gives a result that is valid only as seen from one link end. To obtain a correct and general double-directional spectrum, at least double-scattering processes would have to be modeled.
}

environments. Typical values can be obtained from the maps of the virtual deployment areas (see below). Similarly, the rate of occurrence of transitions, and their duration, can be obtained from the maps of the virtual deployment areas and the prescribed route (including velocity) of the MS. Note that the transition function is chosen to be the same for all MPCs, regardless of their angle of arrival. This is a somewhat arbitrary simplification in the model.

A more abstract approach would be the definition of the probability of transitions as a Markov process. This presumes that the probability of changing from one RE to another depends only on what RE one is currently in, but not on the long-term history. While this is not exact, it seems like a reasonable approximation for most applications. However, this approach would require much information about typical city structures, as well as mobility models. We have thus decided that this would not facilitate ease in application, as required by the objectives of the work reported herein.

\section{Transition Between Cell Types}

Since handovers between macro- and microcells or microand picocells are likely to occur in practice, interoperability between different cell types also has to be considered. Related questions occur with respect to operation in microcells with the MS indoors and in picocells with the MS outdoors, situations which correspond to scenarios that exist immediately before/after a handover.

For transitions between cell types no extra functionality is required because a transition between different cell types always goes in parallel with a change of REs. That is, if a transition between cell types is required, a global channel update is performed with new parameters chosen from the subset defined for the target cell type. This transition can be made smooth by applying appropriate transition functions as explained above; the same cautionary notes as in Sec. IV.B about the validity of the transition function apply also here.

\section{Clustering}

Propagation measurement results show that in some REs the MPCs are not always uniformly spread in the $(\tau, \Omega)$-space but typically arrive in clusters [50], [49], [51], [52], [53]. In outdoor environments, clustering mostly appears because the MPCs are created by the interaction of the TX signal with objects located in a certain region (IO clusters), ${ }^{16}$ like a group of high-rise buildings. A cluster can also arise by the interaction of a "local IO cluster" (objects close to the MS) either with a LOS wave or a wave arising from a single (specular) reflection from another object (e.g., a single high-rise building with a smooth surface). In indoor NLOS scenarios, a clustering pattern arises due to openings such as doorways or due to angular spreading at wall transmissions (Any plane wave is spread in angle when the wall through which it is transmitted is not an ideal, (possibly layered), plane-parallel dielectric [54]). Moreover, when energy of an IO cluster can reach the RX not only on a "direct path," but also by a reflection on a nearby wall, we see additional clusters,

\footnotetext{
${ }^{16}$ In the following, we denote as "cluster" the multipath components grouped together in the $(\tau, \Omega)$ plane. The group of physical objects located in a certain region is called "IO cluster."
} 
since the reflected components have strongly differing angles of incidence. Thus, clustering can be brought about by the physical propagation process in the sense that components of a cluster experience the same large-scale behavior, while the large-scale behavior for components of distinct clusters is independent.

1) Definition: Formally, the indices of the MPCs $h_{\ell}(\vec{r}, \tau, \Omega), \ell=1, \ldots, L$ can be grouped into $M \leq L$ disjoint classes (or clusters)

$$
C_{1}, \ldots, C_{M},
$$

where each class has $N_{m} \geq 1$ elements, and

$$
\sum_{m=1}^{M} N_{m}=L \text {. }
$$

With this notation, (4) can be re-written as

$$
h(\vec{r}, \tau, \Omega)=\sum_{m=1}^{M} \sum_{n \in C_{m}} h_{n}(\vec{r}, \tau, \Omega) .
$$

Physically, a cluster is a group of MPCs with similar $\tau$, $\Omega$. In macro-cells, clustering usually occurs whenever there are large objects like buildings or hills that cause significant power to reach the MS from slightly different directions and with slightly different excess delays, e.g., [50]. In micro- and picocells, also other processes like waveguiding can also often lead to clustering. It is important to note that it is not possible to give a mathematically unique definition of a cluster from one measurement, or even from a series of measurements in a small area. Visual inspection of PDDPs has been reported in previous papers, e.g., [34], [52] for identifying clusters. Alternatively, clusters are defined in such a way that MPCs of a cluster must exhibit the same large-scale behavior, e.g. largescale fading, changes in the direction-of-arrival and runtime, etc.

It is important to note that a "fading multipath group," i.e., a number of MPCs that are indistinguishable to a RX because of limited resolution, is different from a cluster. A cluster consists usually of several multipath groups with similar delays and angles, and is surrounded (in the delay-angle plane) by areas of no "significant" power. ${ }^{17}$ Consequently, the MPCs belonging to a cluster do not change, even as the resolution of the measurement device becomes finer and finer; while the MPCs belonging to a multipath group change as the resolution becomes finer.

The above description of clustering was formal, and does not yet explain why the identification of clusters should simplify the modeling process. Simplifications arise because the parameters of a cluster, e.g., the normalized small-scale PDDP, do not change with time. To give an example, the PDP of a single cluster is always an exponential function. When the PDDP consists of three clusters, we have a total of three exponentials. When the MS moves over large areas, the position of the exponentials relative to each other changes, but the shape of the cluster PDP remains unchanged. In addition, the cluster PDDP can be written as a product of a cluster PDP

\footnotetext{
${ }^{17}$ For a receiver with very low angular/delay resolution, it might happen that each cluster contains only a single multipath group, or even that a multipath group contains several clusters.
}

and a cluster PAS, ${ }^{18}$ while it is not possible to write the total PDDP in such a multiplicative way.

2) Visibility Regions: In order to model the appearance and disappearance of clusters, the COST259 DCM introduces the concept of "Visibility Regions." For each cluster, we define certain physical regions in a coverage area so that if the MS is in such a region, the cluster is active, i.e., the MPCs belonging to that cluster contribute to the PDDP, otherwise they are not. ${ }^{19}$ Note that this description mixes concepts of geometry and channel impulse responses functions: a cluster of MPCs (in the PDDP) is present if the MS is in a certain geometric region! ${ }^{20}$ The pdf of the location of visibility regions within specific REs is specified in Papers II and III. ${ }^{21}$ For each cluster, a separate set of visibility regions must be generated. The maximum number of clusters must be chosen in such a way that (averaged over all MS positions in the cell) the mean number of active clusters equals the cluster number specified for the RE. A more detailed explanation of the visibility regions is given in Paper II, as the concept is mainly used in association with clusters in macrocells.

For picocells, a somewhat different approach is used (see also Paper III): the generation and elimination (birth and death) of the MPCs is modeled as a Poisson process, with given rates for the creation and life duration. The mean of the number of MPCs at any given time is as the ratio between the creation rate and the death rate. Theoretically, the number of MPCs can drop to zero, since no minimum number is prescribed. However, since the average number is around 15 , such a case would be exceedingly rare. ${ }^{22}$

\section{E. The Virtual Deployment Area}

In macrocell environments, we define all parameters statistically. For micro- and picocells we choose a slightly different approach in which we use what we call Virtual Deployment Areas (VDAs). In this approach, we define a street map or office map that describes a typical micro- or picocell environment. The positions and dimensions of the streets or offices are described deterministically. By defining such a virtual environment, parameters like the occurrence rate of street crossings or transition lengths can be derived, depending on a chosen mobile route.

The layouts chosen for VDAs are considered fairly typical, according to the experience of the participants of COST259; the exact specifications are given in Paper III. However, as each person tends to judge as "typical" the environments

\footnotetext{
${ }^{18}$ This statement is not exact, as, e.g., at very small delays of the first cluster, waves must come from a narrow angular range. Still, measurements have shown that such a multiplicative approach is a good approximation in many cases, see also Papers II and III.

${ }^{19}$ This would lead to a hard onset of the visibility of the cluster. For actual implementation, we use a soft onset, with a transition function related to the transition functions of radio environments, see Paper II.

${ }^{20}$ Note that the concept can also be interpreted in a completely geometrical fashion: if the MS is in the visibility region, it has LOS (or at least quasiLOS) to the IO cluster that gives rise to the (MPC) cluster. However, this interpretation is only valid for the case that clusters are created by singleinteraction processes.

${ }^{21}$ Note that the probability for LOS is treated also with the help of visibility regions, and actually uses a nonuniform distribution of visibility regions, see Paper II.

${ }^{22}$ Furthermore, it would not contradict physical reality, since this would just be loss of connection.
} 
he/she is most familiar with, a definite determination of "what is typical" would require the statistical evaluation of building plans of many houses, office buildings, etc. At the moment (and probably far into the future), such an undertaking is not possible, or at least the possible gain does not justify the enormous expenses required. However, rather than using VDAs defined in Paper III, a user can specify his own for testing related to specific environments.

\section{F. The Number of Multipath Components}

The number of MPCs that has to be accounted for in the model is another important topic. The following considerations have to be taken into account:

1) For the specification of the model, it is necessary to investigate the number of fading MPGs (taps in a tappeddelay-line model) that has to be included. As we increase the temporal or spatial resolution, we might find that for many possible delays or directions, there are no incident waves at all. The COST259 DCM specifies the PDDP $P(\tau, \Omega)$, which can be written as $p_{P}(P \mid \tau, \Omega) p_{\tau, \Omega}(\tau, \Omega)$. Here, $p_{\tau, \Omega}(\tau, \Omega)$ is the probability that a wave occurs with delay $\tau$ and direction $\Omega . p_{P}(P \mid \tau, \Omega)$ is the power of a wave that is actually coming from that direction. If the resolution of the receiving system is poor, the details of this factorization have no effect, as there will always be waves within any delay/angle bin. However, at high resolution, this might not be the case.

2) The COST259 DCM gives a small-scale averaged PDDP and assumes that within each delay/angle bin, the amplitude is Rayleigh fading. ${ }^{23}$ However, as the delay or angular resolution increases, fewer and fewer MPCs contribute to each bin, so that the basic assumptions for Rayleigh fading (large number of equally powered echoes) are no longer fulfilled and the fading within each bin begins to behave in accordance with other models, such as the Nakagami distribution [55].

The COST259 DCM does not specify any recommendations for the modeling of the amplitude fading for very fine resolution, but just states that the model should be implemented such that there is Rayleigh fading. We also stress that the validity region is postulated, rather than based on measurements, due to a lack of extensive measurement campaigns reporting amplitude distributions from measurements with different bandwidths.

\section{Summary And ReCOMMENDATIONS FOR FUTURE WORK}

In this paper, we have reported concepts that form the basis of the COST259 DCM. Due to the different scales on which the physical processes happen, a multi-layer approach is taken. First, we subdivide macro- micro- and picocells by morphological characteristics, giving a total of 13 radio environments. Each radio environment is characterized by external parameters and global parameters, which define the propagation characteristics of a whole cell. Global parameters could be defined by specifying pdfs, and one realization drawn

\footnotetext{
${ }^{23}$ Apart from the bin containing the LOS component, which might be Rice fading.
}

from these pdfs would yield a set of local parameters. The local parameters, in turn, define models for the propagation characteristics in a small area (approx. 10 wavelengths), and this in turn, allows the generation of instantaneous DCIRs.

While the COST259 DCM is fairly general, there are some specific assumptions that restrict its applicability, which should be investigated in further research. These include:

- an assumption of stationary IOs: the model assumes that the IOs are stationary, and that all temporal variations originate from movement of the MS. This assumption is not strictly true in practice; the error made through its use depends on the environment under study. For mobile handsets and other devices that would be used close to the body of a user, the impact of the user, and his/her movements relative to the mobile device, should be taken into account. Furthermore, the movement of other people and cars should be taken into account. Experimental results applicable for indoor wireless Local Area Networks (LANs) [56] and for outdoor fixed wireless systems [35] indicate that the Rice factor typifying Rician fading, as a result of moving IOs can be as low as $10 \mathrm{~dB}$. For other environments and scenarios, we are not aware of any experimental investigations. In order to take the effect into account in future models, extensive measurements are required to enable quantification of this effect in different environments. Furthermore, new modeling methods will be required. Using an "equivalent" movement of the MS to emulate movement of IOs, while simple, is not physically justified. Geometrical modeling thus seems more promising for including the separate modeling of MS and IOs that is required if their separate motions are to be represented.

- an assumption of complex Gaussian delay tap statistics: the model assumes that the amplitude statistics of a tapped delay line representation of a channel impulse response function are complex Gaussian. This in turn requires that the number of MPCs in each resolvable multipath group is sufficiently high that the central limit theorem is applicable. Further experimental investigations will have to be performed to analyze the validity of this assumption in different environments, and for systems with different bandwidths. For example, several outdoor measurements [57] indicate that the assumption might be violated for bandwidths above $5 \mathrm{MHz}$. Recent measurements in an industrial environment [58] however found the assumption to be valid even for bandwidths in excess of $1 \mathrm{GHz}$.

- an assumed function for changes in the shadowing during the transition function between environments: the model makes the simplifying assumption that the shadowing that governs the transition from one environment to the next is governed by a single transition function that is independent of the angle of the arriving MPC. This assumption is an oversimplification used for computational convenience. As far as we know, there are no experimental investigations on that topic yet. Future work should investigate whether using different Fresnel parameters for each MPC, depending on their directions at the MS, leads to a significant improvement in accuracy.

Despite these issues, the model framework described in this 
paper allows a fairly general representation of mobile radio channels and includes many effects that have hitherto been ignored in standardized channel models. The parameterization of the model, and results for different environments, are described in the companion papers (Papers II and III).

\section{ACKNOWLEDGMENT}

We thank all other participants of the COST259 subgroup 2.1, especially I. de Costa, T. Englert, D. Hampicke, P. Karlsson, R. Kattenbach, N. Lohse, J. Talvitie, and P. Vainikainen. Their ideas significantly influenced the work presented here. We also thank the chairman of WG2, Prof. Ernst Bonek, and the Chairman of COST259, Prof. Luis Correia. The financial support of the European Union for the COST259 action is gratefully acknowledged. We also thank the anonymous reviewers, whose suggestions have greatly helped to improve the manuscript. We are especially grateful to Dr. Robert Bultitude, whose detailed and constructive suggestions contributed enormously to this paper.

\section{REFERENCES}

[1] M. Mouly and M. B. Pautet, "The GSM system for mobile communications," 1992.

[2] M. Failli, ed., Digital Land Mobile Radio Communications-COST 207. Luxemberg: European Union, 1989.

[3] M. Hata, "Empirical formulas for propagation loss in land mobile radio service," IEEE Trans. Veh. Technol., vol. 29, no. 3, pp. 317-325, Aug. 1980.

[4] W. C. Y. Lee, Mobile Communications Engineering. New York: McGrawHill, 1982.

[5] E. Damosso, ed., Digital Mobile Communications - The View of COST 231. Commission of the European Union, 1998.

[6] "Guidelines for evaluation of radio transmission technologies for IMT2000," International Telecommunications Union, Tech. Report, 1997.

[7] J. Medbo and P. Schramm, "Channel models for HIPERLAN/2," ETSI/BRAN document no. 3ERI085B, Tech. Report, European Telecommunications Standards Institute, 2000.

[8] T. Ojanpera and R. Prasad, Wideband CDMA for Third Generation Mobile Communications. Norwood, MA: Artech House Publishers, 1998.

[9] R. Kattenbach, "Statistical modeling of small-scale fading in directional radio channels," IEEE J. Select. Areas Commun., vol. 20, no. 3, pp. 584592, Apr. 2002.

[10] J. B. Liberti and T. S. Rappaport, "A geometrically based model for line-of-sight multipath radio channels," in Proc. IEEE Vehicular Technol. Conf., May 1996, vol. 2, pp. 844-848.

[11] P. Petrus, J. H. Reed, and T. S. Rappaport, "Geometrical-based statistical macrocell channel model for mobile environments," IEEE Trans. Commun., vol. 50, no. 3, pp. 495-502, Mar. 2002.

[12] A. A. M. Saleh and R. R. Valenzuela, "A statistical model for indoor multipath propagation," IEEE J. Select. Areas Commun., vol. 5, no. 2, pp. 128-137, Feb. 1987.

[13] Q. Spencer, M. Rice, B. Jeffs, and M. Jensen, "Indoor wideband time/angle of arrival multipath propagation results," in Proc. IEEE Vehicular Technol. Conf., May 1997, vol. 3, pp. 1410-1414.

[14] R. M. Buehrer, S. Arunachalam, K. H. Wu, and A. Tonello, "Spatial channel model and measurements for IMT-2000 systems," in Proc. IEEE 53rd Vehicular Technol. Conf., May 2001, vol. 1, pp. 342-346.

[15] J. Kim and W. Chung, "A spatio-temporal channel model for position location techniques via AOA and TDOA," in Proc. IEEE 53rd Vehicular Technol. Conf., May 2001, vol. 1, pp. 233-237.

[16] K. I. Pedersen, P. E. Mogensen, and B. H. Fleury, "Spatial channel characteristics in outdoor environments and their impact on bs antenna system performance," in Proc. IEEE 48th Vehicular Technol. Conf., May 1998, vol. 2, pp. 719-723.

[17] A. Abdi and M. Kaveh, "A space-time correlation model for multielement antenna systems in mobile fading channels," IEEE J. Select. Areas Commun., vol. 20, no. 3, pp. 550-560, Apr. 2002.

[18] O. Norklit and J. B. Andersen, "Diffuse channel model and experimental results for array antennas in mobile environments," IEEE Trans. Antennas Propagat., vol. 46, no. 6, pp. 834-840, June 1998.
[19] R. J. Piechocki, J. P. McGeehan, and G. V. Tsoulos, "A new stochastic spatio-temporal propagation model (SSTPM) for mobile communications with antenna arrays," IEEE Trans. Commun., vol. 49, no. 5, pp. 855-862, May 2001.

[20] Y. Oda, K. Tsunekawa, and M. Hata, "Geometrically based directional channel model for urban mobile communication systems," in Proc. IEEE Conf. Antennas \& Propagation for Wireless Commun., Nov. 2000, pp. 8790.

[21] M. G. Marques and L. M. Correia, "A wideband directional channel model for umts microcells," in Proc. 12th IEEE Int. Symp. on Personal, Indoor and Mobile Radio Communications, Sep. 2001, vol. 1, pp. B122B126.

[22] R. J. C. Bultitude, C. D. Charalambous, X. Li, M. Herben, and G. Brussaard, "Development of a model for realistic portrayal of random time variations on mobile radio channels," in Proc. URSI General Assembly, Aug. 2002, p. 2119.

[23] A. F. Molisch, H. Asplund, M. Steinbauer, and N. Mehta, "Backward compatibility of the cost 259 directional channel model," in Proc. WPMC, Oct. 2002, pp. 549-553.

[24] H. Asplund, A. A. Glazunov, A. F. Molisch, K. I. Pedersen, and M. Steinbauer, "The COST259 directional channel model II-Macrocells," IEEE Trans. Wireless Commun., to appear.

[25] A. F. Molisch, J. E. Dietert, R. Heddergott, M. Steinbauer, and T. Zwick, "The COST259 directional channel model III-Micro- and picocells," IEEE Trans. Wireless Commun., to be submitted.

[26] P. Bello, "Characterization of randomly time-variant linear channels," IEEE Trans. Commun., vol. 11, no. 4, pp. 360-393, Dec. 1963.

[27] B. H. Fleury, "First- and second-order characterization of direction dispersion and space selectivity in the radio channel," IEEE Trans. Inform. Theory, vol. 46, no. 6, pp. 2027-2044, Sep. 2000.

[28] J. H. Hudson, Adaptive Array Principles. London: Peter Peregrinus Ltd., 1981.

[29] R. A. Kennedy, T. D. Abhayapala, and D. B. Ward, "Broadband nearfield beamforming using a radial beampattern transformation," IEEE Trans. Signal Processing, vol. 46, no. 8, pp. 2147-2156, Aug. 1998.

[30] M. Steinbauer, "The Radio Propagation Channel-A Non-Directional, Directional, and Double-Directional Point-of-View." PhD thesis, Technical University, Vienna, 2000.

[31] R. Heddergott, "Stochastic Model of the Mobile Radio Channel Considering Delay and Angle Dispersion in Indoor Environments" (in German). $\mathrm{PhD}$ thesis, ETH, Zurich, 2001.

[32] R. Bultitude, "Statistical modelling of mobile radio channels for wideband applications," in Proc. XXVIth URSI General Assembly, Aug. 1999, p. ECR-1 b5.

[33] Y. L. C. DeJong and M. H. A. J. Herben, "Prediction of local mean power using 2-D ray-tracing-based propagation models," IEEE Trans. Veh. Technol., vol. 50, no. 1, pp. 325-331, Jan. 2001.

[34] L. Vuokko, P. Vainikainen, and J. Takada, "Clusterization of measured direction-of-arrival data in an urban macrocellular environment," in Proc. IEEE Int. Symp. on Personal, Indoor and Mobile Radio Communications, Sep. 2003, vol. 2, pp. 1222-1226.

[35] V. Erceg, D. G. Michelson, S. S. Ghassemzadeh, L. J. Greenstein, A. J. Rustako, Jr., P. B. Gerlain, M. K. Dennison, R. S. Roman, D. J. Barnickel, S. C. Wang, and R. R. Miller, "A model for the multipath delay profile of fixed wireless channels," IEEE J. Select. Areas Commun., vol. 17, no. 3, pp. 399-410, Mar. 1999.

[36] P. A. Bello and B. Nelin, "Predetection diversity combining with selectively fading channels," IEEE Trans. Commun., vol. 10, no. 1, pp. 32-42, Mar. 1962.

[37] G. L. Turin, F. D. Clapp, T. L. Johnston, S. B. Fine, and D. Lavry, "A statistical model of urban multipath propagation," IEEE Trans. Veh. Technol., vol. 21, pp. 1-9, Jan. 1972.

[38] T. Zwick, C. Fischer, D. Didascalou, and W. Wiesbeck, "A stochastic spatial channel model based on wave propagation modeling," IEEE $J$. Select. Areas Commun., vol. 18, pp. 6-15, Jan. 2000.

[39] B. Fleury, U. P. Bernhard, and R. Heddergott, "Advanced radio channel model for Magic WAND," in Proc. ACTS Mobile Communications Summit, Nov. 1996, vol. II, pp. 600-607.

[40] R. Heddergott, U. P. Bernhard, and B. H. Fleury, "Stochastic radio channel model for advanced indoor mobile communication systems," in Proc. IEEE 8th Int. Symp. on Personal, Indoor and Mobile Radio Communications, Sep. 1997, vol. 1, pp. 140-144.

[41] J. Fuhl, A. F. Molisch, and E. Bonek, "Unified channel model for mobile radio systems with smart antennas," in Proc. IEE Radar, Sonar and Navigation, Feb. 1998, vol. 145, pp. 32-41.

[42] P. A. Bello, "Characterization of randomly time-variant linear channels," IEEE Trans. Commun., vol. 11, no. 4, pp. 360-393, Dec. 1963.

[43] R. Kattenbach, "Charakterisierung Zeitvarianter Indoor-Funkkanäle an- 
hand ihrer System- und Korrelationsfunktionen.” $\mathrm{PhD}$ thesis, Universität Gesamthoschschule Kassel, Kassel, Germany, 1997.

[44] J. J. Blanz, P. W. Baier, and P. Jung, "A flexibly configurable statistical channel model for mobile radio systems with directional diversity," in Proc. 2nd ITG Fachtagung Mobile Kommunikation, Sep. 1995, pp. 93100.

[45] A. F. Molisch, A. Kuchar, J. Laurila, K. Hugl, and E. Bonek, "Efficient implementation of a geometry-based directional model for mobile radio channels," in Proc. Vehicular Technol. Conf., Sep. 1999, vol. 3, pp. 14491453.

[46] P. Eggers, "Generation of base station DOA distributions by Jacobi transformation of scattering areas," IEEE Electron. Lett., vol. 34, no. 1, pp. 24-26, Jan. 1998.

[47] A. F. Molisch, J. Laurila, and A. Kuchar, "Geometry-base stochastic model for mobile radio channels with directional component," in Proc. 2nd Intelligent Antenna Symp., Sep. 1998.

[48] A. Papoulis, Probability, Random Variables, and Stochastic Processes. New York: McGraw-Hill, 1965.

[49] A. Kuchar, J. P. Rossi, and E. Bonek, "Directional macro-cell channel characterization from urban measurements," IEEE Trans. Antennas Propagat., vol. 48, no. 2, pp. 137-146, Feb. 2000.

[50] U. Martin, "Spatio-temporal radio channel characteristics in urban macrocells," in Proc. IEE Radar, Sonar and Navigation, Feb. 1998, vol. 145 , no. 1 , pp. $42-49$.

[51] P. Pajusco, "Experimental characterization of DOA at the base station in rural and urban area," in Proc. IEEE Vehicular Technol. Conf., May 1998, vol. 2, pp. 993-997.

[52] M. Toeltsch, J. Laurila, K. Kalliola, A. F. Molisch, P. Vainikainen, and E. Bonek, "Statistical characterization of urban spatial radio channels," IEEE J. Select. Areas Commun., vol. 20, no. 3, pp. 539-549, Apr. 2002.

[53] T. Zwick, C. Fischer, and W. Wiesbeck, "A stochastic multipath channel model including path directions for indoor environments," IEEE J. Select. Areas Commun., vol. 20, no. 6, pp. 1178-1192, Aug. 2002.

[54] H. L. Bertoni, Radio Propagation for Modern Wireless Systems. Upper Saddle River, NJ: Prentice Hall, 2000.

[55] D. Cassioli, M. Z. Win, and A. F. Molisch, "The ultra-wide bandwidth indoor channel: From statistical model to simulations," IEEE J. Select. Areas Commun., vol. 20, no. 6, pp. 1247-1257, Aug. 2002.

[56] J. Medbo, F. Harryson, and J. E. Berg, "Temporal radio channel variations with stationary terminal," in Proc. IEEE Vehicular Technol. Conf., Sep. 2004, vol. 1, pp. 91-95.

[57] V. Adnani, R. J. C. Bultitude, and R. Hafez, "Propagation-measurement based rake receiver BER performance predictions for WCDMA links operating in urban microcellular environments," in Proc. U.R.S.I General Assembly, p. CBA.7(01060).

[58] J. Karedal, S. Wyne, P. Almers, F. Tufvesson, and A. F. Molisch, "Statistical analysis of the UWB channel in an industrial environment," in Proc. IEEE Vehicular Technol. Conf., Sep. 2004, vol. 1, pp. 26-29.

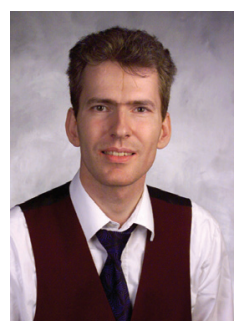

Andreas Molisch (S'89-M'95-SM'00-F'05) received the Dipl. Ing., Dr. techn., and habilitation degrees from the Technical University Vienna (Austria) in 1990, 1994, and 1999, respectively. From 1991 to 2000, he was with the TU Vienna, becoming an associate professor there in 1999. From 20002002, he was with the Wireless Systems Research Department at AT\&T (Bell) Laboratories Research in Middletown, NJ. Since then, he has been a Senior Principal Member of Technical Staff with Mitsubishi Electric Research Labs, Cambridge, MA. He is also professor and chairholder for radio systems at Lund University, Sweden.

Dr. Molisch has done research in the areas of SAW filters, radiative transfer in atomic vapors, atomic line filters, smart antennas, and wideband systems. His current research interests are MIMO systems, measurement and modeling of mobile radio channels, and UWB. Dr. Molisch has authored, co-authored or edited four books (among them the recent textbook Wireless Communications, Wiley-IEEE Press), eleven book chapters, ninety journal papers, and numerous conference contributions.

Dr. Molisch is an editor of the IEEE TRAns. Wireless Comm., co-editor of a recent special issue on MIMO and smart antennas in J. Wireless Comm. Mob. Comp., and co-editor of a recent JSAC special issue on UWB. He has been member of numerous TPCs, vice chair of the TPC of VTC 2005 spring, and will be general chair of ICUWB 2006. He has participated in the European research initiatives "COST 231," "COST 259," and "COST273," where he was chairman of the MIMO channel working group, and is also chairman of Commission C (signals and systems) of URSI (International Union of Radio Scientists). Dr. Molisch is a Fellow of the IEEE and recipient of several awards.
Henrik Asplund (M'05) received the M.Sc. degree from Uppsala University, Sweden in 1996. Since then he has been with the Antenna Systems and Propagation Research Group at Ericsson Research, Stockholm, Sweden. His current research interests are measurement and modeling of mobile radio channels, antenna diversity, smart antennas, and MIMO systems. Henrik Asplund has participated in the European research initiatives "COST 259" and "COST 273."

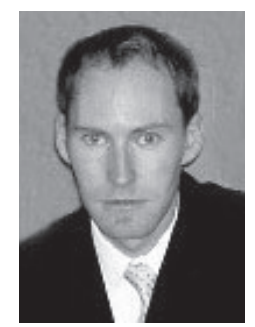

Martin Steinbauer (S'99-M'02) received his Dipl.Ing. (1996) and Doctoral Degree (2001), both with distinction, from Vienna University of Technology, Austria (TU-Wien). He received two performance scholarships. After his diploma thesis which he conducted for the German mobile network operator E-Plus GmbH., Düsseldorf, he was research assistant at the Institute of Communications and Radio Frequency Engineering at TU-Wien. From 2002 to first half of 2005 he was with the Network Planning division at mobilkom austria AG\&CoKG, Vienna. In 2005, he joined the Group Technology division within mobilkom austria group services $\mathrm{GmbH}$.

Dr. Steinbauer has done research in the field of mobile radio measurement and modeling for simulation, wideband non-stationary channel characterization, clustering effects, and signal processing for MIMO channel estimation $\mathrm{He}$ did pioneer work in MIMO measurements that he conducted together with TU-Ilmenau. Dr. Steinbauer participated in the EU projekt METAMORP and was chairman of the subworking group on directional channel modeling within the European research initiative COST259. He authored or co-authored several publications in the field of mobile radio.

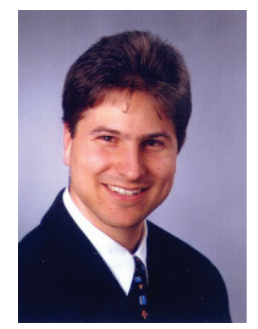

Thomas Zwick (S'95-M'00-SM'06) received the Dipl.-Ing. (M.S.E.E) and the Dr.-Ing. (Ph.D.E.E) degrees from the Universität Karlsruhe (TH), Germany in 1994 and 1999, respectively. From 1994-2001 he was research assistant at the Institut für Höchstfrequenztechnik und Elektronik (IHE) at the Universität Karlsruhe (TH), Germany. Between February 2001 and September 2004 he was Research Staff Member at the IBM T. J. Watson Research Center in Yorktown Heights, NY USA. Since October 2004 T. Zwick is with Siemens AG, Lindau, Germany.

His research topics include wave propagation, stochastic channel modeling, channel measurement techniques, material measurements, microwave techniques, millimeter wave antenna design, wireless communication and radar system design. He participated as an expert in the European COST231 Evolution of Land Mobile Radio (including Personal) Communications and COST259 Wireless Flexible Personalized Communications. For the Carl Cranz Series for Scientific Education, he served as a lecturer for Wave Propagation. He received the best paper award on the Intern. Symp. on Spread Spectrum Techn. and Appl. ISSTA 1998. In 2005 he received the Lewis award for outstanding paper at the IEEE International Solid State Circuits Conference. 